\title{
Hypophysentumor wegen Kommafehler
}

\author{
Bei einer 37-jährigen Frau war seit 20 \\ Jahren eine primäre Hypothyreose \\ bekannt. Nun wurde die Patientin \\ wegen eines Makroadenoms der \\ Hypophyse zum Endokrinologen \\ überwiesen.
}

- Vor neun Monaten hatte ein TSHWert von 7,38 IU/l zu einer Erhöhung der Substitutionsdosis von L-Thyroxin von 0,2 auf $0,25 \mathrm{mg}$ täglich geführt. Trotz dieser Dosiserhöhung fühlte sich die Patientin zunehmend müde und litt unter Kopfschmerzen, Ausfluss aus den Brustwarzen und Gewichtszunahme. An Nebendiagnosen bestanden eine Hypertonie, eine Gicht, eine bipolare Störung und eine Arthrose. Die übrige Medikation bestand aus Atenolol, Colchicin, Quetiapin, Bupropion, Escitalopram, Pregabalin, Topiramat und Hydroxychloroquin. Vitamine und pflanzliche Präparate nahm sie nicht ein.

Bei der Untersuchung bot die Patientin alle Zeichen der Hypothyreose mit trockener Haut und verzögerten Reflexen. Im Labor fiel vor allem ein TSH von über $100 \mathrm{IU} / \mathrm{l}$ auf bei etwas erniedrigten Werten für $\mathrm{fT}_{3}$ und $\mathrm{fT}_{4}$. Auch das Prolaktin war mit $79 \mu \mathrm{g} / \mathrm{l}$ geringfügig erhöht. Im MRT erwies sich die Hypophyse als homogen vergrößert, weswegen man eine Hyperplasie annahm (Abb.).

Die Dosis von L-Thyroxin wurde auf 0,3 mg täglich erhöht. Im Lauf der nachfolgenden Monate besserten sich die Symptome der Patientin zunehmend und sämtliche Laborbefunde normalisierten sich. Auch die Größe der Hypophyse war rückläufig, wie ein KontrollMRT ergab (Abb.). Weitere Nachfor-

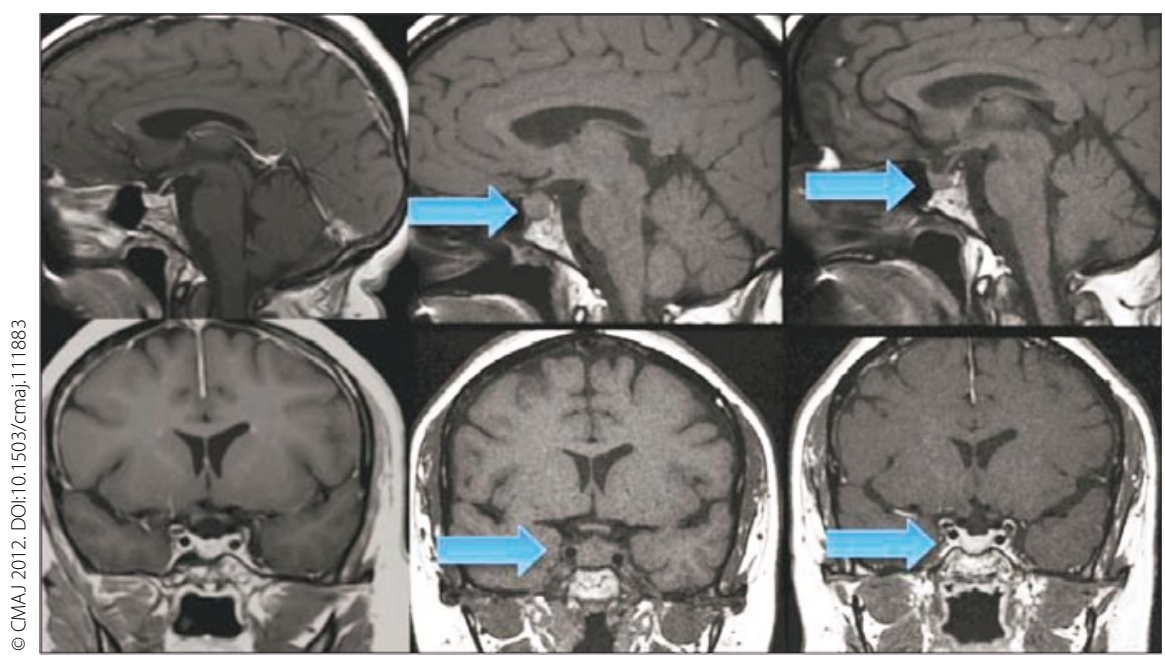

MRT des Kopfes der 37-jährigen Frau (Sagittal- und Koronarschnitte) bei der Erstuntersuchung (Bilder links), bei der Vorstellung beim Endokrinologen mit homogener Vergrößerung der Hypophyse (Pfeile, mittlere Bilder) und nach mehrmonatiger suffizienter Substitutionstherapie mit L-Thyroxin (Bilder rechts).

schungen darüber, wie es zu diesem merkwürdigen Verlauf kommen konnte, fanden eine simple Lösung. Als der Hausarzt der Patientin die L-ThyroxinDosis auf $0,25 \mathrm{mg}$ täglich erhöht hatte, irrte sich die Apotheke um eine Kommastelle und gab tatsächlich nur 0,025 mg täglich aus. Wie es zu diesem Fehler kommen konnte, bleibt rätselhaft.

\section{Kommentar}

Eine schwere primäre Hypothyreose kann zu einer Hyperplasie der Hypophyse führen, wenn das Organ durch TRH überstimuliert wird. Gleichzeitig kann eine vermehrte Sekretion von Prolaktin auftreten. Nachdem bildgebende Verfahren nicht zuverlässig zwischen einer Hyperplasie und einem Makroadenom unterscheiden können, bedarf es einer sorgfältigen klinischen Untersuchung und einer kritischen Prüfung der Symptomatik. Derartige Irrtümer in der Apotheke dürften eher die Ausnahme sein. Viel häufiger liegt das Problem in einer Non-Compliance der Patienten.

Fabula docet: Handschriftlich ausgefüllte Rezepte haben gerade bei den notorisch unleserlichen Handschriften von Ärzten ihre Tücken. Mit der allgemeinen Verbreitung von elektronisch ausgefüllten Rezepten sollte das aber bald kein Problem mehr sein.

H. S. FÜESSL =

\footnotetext{
- K. Clemens, S. van Uum

(Korres.: Dr. Stan Van Uum, stan.vanuum@ sjhc.london.on.ca): A pituitary mass as consequence of a decimal error in levothyroxine dose. CMAJ 2012. DOI:10.1503/cmaj.111883
} 